\title{
Nutzungsmuster von Internet und Computerspielen
}

\author{
Ergebnisse einer Beobachtungsstudie an Tiroler Jugendlichen
}

David Riedl · Andrea Stöckl · Charlotte Nussbaumer · Gerhard Rumpold · Kathrin Sevecke · Martin Fuchs

Eingegangen: 27. Juni 2016 / Angenommen: 22. Oktober 2016 / Online publiziert: 8. November 2016

(c) Der/die Autor(en) 2016. Dieser Artikel ist eine Open-Access-Publikation.

\section{Zusammenfassung}

Grundlagen Der Gebrauch von digitalen Medien wie Internet und Computerspielen ist vor allem in der Gruppe von Jugendlichen so stark angestiegen, dass in der westlichen Welt nahezu alle jungen Menschen mehr oder weniger regelmäßig einschlägige Technologien gebrauchen. Vor diesem Hintergrund werden seit einigen Jahren auch Nutzungsformen mit möglichen schädlichen Auswirkungen auf Jugendliche erfasst. Ziel unserer Studie war deshalb, die Prävalenz von pathologischem Mediengebrauch in einer Stichprobe von Tiroler Jugendlichen zu erheben.

Methodik 398 SchülerInnen (mittleres Alter: 15,2 \pm 2,3 Jahre, 34,2\% weiblich) wurden mittels strukturierter Fragebögen zum Internetnutzungs- und Computerspielverhalten sowie zur Selbstwirksamkeit befragt. Ergebnisse Im Einklang mit bisherigen Studien zeigte sich in unserer Stichprobe eine Prävalenz von 7,7 \% für problematischen Internet-Gebrauch sowie von 3,3\% für suchtartigen Internet-Gebrauch (CIUS). 5,4\% der untersuchten SchülerInnen zeigten suchtartiges Computerspielverhalten (CSV-S). Als wichtigster Einflussfaktor beeinflusste das Geschlecht der ProbandInnen signifikant die Ergebnisse: Intensivnutzer in den Bereichen Internet und Computerspiele waren häufiger junge Männer, junge Frauen hingegen zeigten signifikant seltener suchtartigen Computerspiel-Gebrauch. Schlussfolgerungen Ein signifikanter Teil der befragten Jugendlichen aus der Tiroler Normalbevölkerung

D. Riedl · A. Stöckl · G. Rumpold

Univ.-Klinik für Medizinische Psychologie, Medizinische Universität Innsbruck, Innsbruck, Österreich

C. Nussbaumer $\cdot$ K. Sevecke $\cdot$ Dr. M. Fuchs $(\bowtie)$

Univ.-Klinik für Kinder- und Jugendpsychiatrie, Department

Psychiatrie und Psychotherapie, Medizinische Universität

Innsbruck, Innrain 52, 6020 Innsbruck, Österreich

martin.fuchs@tirol-kliniken.at zeigte Schwierigkeiten in der Entwicklung von kompetenter Mediennutzung. Dies könnte ein Hinweis auf die steigende Bedeutung von Präventionsarbeit, z. B. in Form von Medienpädagogik sein. In einem Folgeprojekt sollen Jugendliche mit psychischen Erkrankungen hinsichtlich ihres Nutzungsverhaltens untersucht und mit der Schulstichprobe verglichen werden.

Schlüsselwörter Internet · Computerspiele · Abhängigkeit · Gebrauch · Jugendliche

\section{Usage patterns of internet and computer games} Results of an observational study of Tyrolean adolescents

\section{Summary}

Background The use of digital media such as the Internet and Computer games has greatly increased. In the western world, almost all young people regularly use these relevant technologies. Against this background, forms of use with possible negative consequences for young people have been recognized and scientifically examined. The aim of our study was therefore to investigate the prevalence of pathological use of these technologies in a sample of young Tyrolean people.

Methods 398 students (average age 15.2 years, SD \pm 2.3 years, $34.2 \%$ female) were interviewed by means of the structured questionnaires CIUS (Internet), CSV-S (Computer games) and SWE (Self efficacy). Additionally, socio demographic data were collected.

Results In line with previous studies, $7.7 \%$ of the adolescents of our sample showed criteria for problematic internet use, 3.3\% for pathological internet use. 5.4\% of the sample reported pathological computer game usage. The most important aspect to influence our results was the gender of the subjects. Intensive users in the field of Internet and Computer games were more often young men, young women, however, showed 
significantly less signs of pathological computer game use.

Conclusions A significant percentage of Tyrolean adolescents showed difficulties in the development of competent media use, indicating the growing significance of prevention measures such as media education. In a follow-up project, a sample of adolescents with mental disorders will be examined concerning their media use and be compared with our schoolsample.

Keywords Internet - Computer games - Addiction · Adolescents

\section{Hintergrund}

Internetfähige Computer und moderne Kommunikationstechnologien haben in private Haushalte Einzug gefunden und sind aus dem Alltag von Kindern, Jugendlichen und Erwachsenen kaum mehr wegzudenken. 1997 nutzten lediglich 6,3\% der befragten 14- bis 19-jährigen deutschen Jugendlichen fallweise das Internet, ab 2010 war diese Nutzungsrate auf $100 \%$ angestiegen und ist seither nicht wieder gesunken. 2015 nutzten bereits über $90 \%$ der befragten Jugendlichen das Internet täglich [1]. In Österreich sind acht von zehn Haushalten mit einer Internetverbindung ausgestattet, womit Österreich über dem europäischen Schnitt liegt. In der Altersgruppe der 16- bis 24-Jährigen benutzten 2013 nahezu $100 \%$ der Österreicher $(99,6 \%)$ das Internet [2]. Eine Nutzungsrate von $100 \%$ konnte auch für deutsche Jugendliche im Alter von 14-19 Jahren gefunden und seit 2010 jährlich repliziert werden [3]. Die Nutzungsrate von mobilem Internet (,zumindest seltener Gebrauch“) in der Altersgruppe der 14- bis 29-Jährigen stieg von 31 \% (2011) auf aktuell $81 \%$ (2015) an. Während 2010 lediglich $9 \%$ der befragten Jugendlichen mobiles Internet als „unverzichtbar“ ansahen, stimmten 2015 bereits fast die Hälfte (48\%) der befragten Jugendlichen dieser Aussage zu. Ortsunabhängig online sein zu können scheint genauso selbstverständlich zu werden, wie unterwegs telefonieren zu können, ohne nach Münzfernsprechern suchen zu müssen [4].

Sowohl auf nationaler wie auch auf internationaler Ebene wurden in den letzten Jahren Befunde repliziert, die zeigen, dass in etwa $20 \%$ aller Kinder und Jugendlichen von psychischen Störungen betroffen sind $[5,6]$. Die weltweit stark ansteigende Nutzungsintensität des Internet vor allem bei Jugendlichen stimulierte einschlägige Forschung bereits relativ früh $\mathrm{zu}$ der Fragestellung, ob auch der Gebrauch neuer Technologien auf pathologische Art und Weise erfolgen könnte, und ob ein solcher „pathologischer Gebrauch von Internet“ (PGI) schädlich für die psychische Gesundheit von jungen Menschen sein könnte. Hinsichtlich der zugrundeliegenden Pathophysiologie wird suchtartiger Internetgebrauch als Verhaltenssucht oder Störung der Impulskontrolle aufgefasst [7,
8]. Im Jahr 2013 wurde „Internet Gaming Disorder“ in den Abschnitt der noch weiter zu beforschenden Störungen des DSM-5 aufgenommen [9], und es ist damit zu rechnen, dass auch im voraussichtlich 2017 erscheinenden ICD-11 eine entsprechende Erwähnung vorkommt. „Internetsucht“ soll dort als eine exzessiv betriebene Verhaltensweise, die Suchtkriterien erfüllt, konzeptualisiert werden [10]. Somit ist derzeit lediglich die problematische Nutzung von internet-basierten Computerspielen durch das DSM5 operationalisiert, im ICD-10 muss das Phänomen mit „F63.9 Abnorme Gewohnheit und Störung der Impulskontrolle, nicht näher bezeichnet“ kodiert werden. Damit stehen weder eine einheitliche Definition noch verbindliche diagnostische Kriterien für PGI zur Verfügung [11].

Neuere Studien sprechen nicht mehr von „Internetsucht“, sondern von „pathologischem Gebrauch von Internet“ $[11,12]$ und wollen damit zum Ausdruck bringen, dass nicht „das Internet“ per se, sondern bestimmte Nutzungsformen schädlich sein könnten. Somit stelle das Internet lediglich das Medium für pathologische Verhaltensweisen dar, und es wäre zweckmäßiger zwischen verschiedenen Anwendungen (bspw. Nutzungsmuster) und deren schädlichem Potential zu differenzieren [13, 14]. Die Wahrscheinlichkeit, ein dysfunktionales Nutzungsverhalten zu zeigen wird sowohl durch Risikofaktoren, aber auch durch protektive Faktoren beeinflusst, wobei neben einem stabilen Selbstwert und Stresstoleranz vor allem eine hohe Selbstwirksamkeitserwartung als protektiver Faktor benannt werden [15].

Bisher veröffentlichte Prävalenzdaten zu PGI zeichnen sich durch eine große Varianz aus. Die einzige verfügbare internationale Metaanalyse, in die 80 Studien mit knapp 90.000 ProbandInnen (Durchschnittsalter 18,4 Jahre) eingeschlossen wurden, zeigte eine mittlere Prävalenzrate von 6,0\% für pathologischen Internetgebrauch. Die Autoren führen die fehlende methodische Einheitlichkeit sowie große regionale Unterschiede als hauptverantwortlich für die großen Schwankungsbreiten an [16]. Im asiatischen Bereich wurden in Studien an Jugendlichen Prävalenzzahlen zwischen 2,4 bis $37,9 \%$ veröffentlicht [17, 18], während amerikanische Studien von Prävalenzraten zwischen 0-26,3\% sprechen [19]. Europäische Studien zeigten ebenfalls inkonsistente Ergebnisse mit Prävalenzzahlen für PGI bis zu 36,7\% [20]. Vor allem in den letzten Jahren jedoch konnten große bevölkerungsbasierte Erhebungen im europäischen Raum verhältnismäßig konsistente Häufigkeitszahlen für problematischen Mediengebrauch zeigen. Durkee et al. [11] veröffentlichten 2012 eine Studie im Rahmen des SEYLE-Projektes, in die 11.956 Jugendliche im Alter zwischen 14-16 Jahren eingeschlossen wurden und fanden bei $4,4 \%$ Hinweise auf PGI. Junge Menschen aus Österreich zeigten mit einer Prävalenz von 3,1\% einen Wert, der etwas unter dem Gesamtdurchschnitt lag, auch in dieser Studie zeigen sich bei 
Tab. 1 Auswahl von Studien zu problematischem Mediengebrauch von Jugendlichen in Europa

\begin{tabular}{|c|c|c|c|c|c|}
\hline Studie & $\begin{array}{l}\text { Untersuchungs-Instrument } \\
\text { (Konstrukt) }\end{array}$ & Stichprobe & $\begin{array}{l}\text { Geschlechterverhältnis } \\
\text { weiblich/männlich in \% }\end{array}$ & Alter & Prävalenz in \% \\
\hline Batthyany et al. 2009 [25] & CSVK-R (PGC) & 1068 & W: 45,2, M: 54,8 & $14,31 \pm 0,82$ & 2,7 \\
\hline Durkee et al. 2012 [11] & YDQ (PGl) & 11.956 & W: 56,29, M: 43,7 & $14,9 \pm 0,98$ & 4,4 \\
\hline Tsitsika et al. 2014 [21] & (PGl) & 13.284 & W: 53, M:47 & $15,8 \pm 0,7$ & 1,2 \\
\hline Blinka et al. 2015 [22] & EIU (PGI) & 18.709 & W: 50,1, M: 49,9 & $13,50 \pm 1,69$ & 1,4 \\
\hline Müller et al. 2015 [24] & AICA-S (IGD) & 12.938 & W: 52,9, M: 47,1 & $15,8 \pm 0,7$ & 1,6 \\
\hline Strittmatter et al. 2015 [23] & YDQ (PGI) & 8807 & W: 55,5, M: 44,5 & $15,0 \pm 1,3$ & 6,73 \\
\hline
\end{tabular}

gleichem Untersuchungsinstrument starke regionale Schwankungen innerhalb der untersuchten Länder [8]. Eine weitere große europäische Studie von Tsitsika et al. [21] fand in $1,2 \%$ der untersuchten Jugendlichen ( $n=13.284$; mittleres Alter 15,8 $\pm 0,7$ Jahre) Hinweise auf PGI. Blinka et al. [22] beschrieben in ihrer Studie eine Prävalenzrate von $1,4 \%(n=18.709$; mittleres Alter $13,50 \pm 1,69$ Jahre). Alle drei Studien benutzten jedoch unterschiedliche Untersuchungsinstrumente. In der bisher einzigen verfügbaren Longitudinalstudie zeigten sich Punktprävalenzzahlen für PGI zwischen 2,7-4,3\% über einen Beobachtungszeitraum von zwei Jahren. Allerdings wiesen lediglich 0,6\% der untersuchten Jugendlichen anhaltend PGI zu allen Untersuchungszeitpunkten auf, was auf eine rasch fluktuierende klinische Symptomatik rückschließen lässt [23].

Müller et al. [24] brachten in einer großen Stichprobe von über 10.000 Jugendlichen zum ersten Mal die neuen DSM-5-Kriterien für „Internet Gaming Disorder" zur Anwendung und konnten für diese Störung eine Prävalenz von 1,6\% unter europäischen Jugendlichen ermitteln.

Ergänzend soll die unseres Wissens einzige weitere österreichische Studie zu problematischem Mediengebrauch erwähnt werden, die bei $2,7 \%$ der untersuchten SchülerInnen Hinweise auf suchtartiges Computerspielen fanden [25].

Tab. 1 zeigt zusammenfassend die oben angeführten großen europäischen Prävalenzstudien zu PGI und IGD sowie die österreichische Erhebung zum Computerspiel-Gebrauch.

Die hier vorgestellte Studie an Tiroler Schülern wurde durchgeführt, um die Prävalenz von pathologischem Mediengebrauch in dieser Altersgruppe mit international gebräuchlichen Untersuchungsinstrumenten zu erfassen. Neben soziodemographischen Daten wurden Faktoren wie Nutzungsdauer, Art der Anwendung, aber auch Selbstwirksamkeit erhoben. Damit soll die Übertragbarkeit von bisher weltweit gewonnenen Studienergebnissen zur Häufigkeit von pathologischem Internet-Gebrauch auf Tiroler Jugendliche überprüft werden. Zusätzlich sollen neben einer reinen Prävalenzschätzung Nutzungsmuster beschrieben werden, die eine differenzierte Betrachtung des Phänomens „pathologischer Mediengebrauch“ ermöglichen. Unseres Wissens nach ist diese Studie die bisher erste dieser Art im österreichischen Raum.

\section{Methodik}

\section{Sample und Datenerhebung}

Die repräsentative Stichprobe umfasst Jugendliche an Innsbrucker Schulen im Alter zwischen 11-18 Jahren. Mittels einer standardisierten Fragebogenbatterie und einem strukturierten Interview wurden die Jugendlichen zwischen November 2011 und März 2012 in Bezug auf ihr Internet- und ComputerspielNutzungsverhalten untersucht. Die Studie wurde in Überstimmung mit der Deklaration von Helsinki geplant und durchgeführt. Da es sich um eine nichtklinische und nicht-interventionelle Feldstudie an einer Schul-Population handelte, wurde die Genehmigung des Innsbrucker Stadtschulrates eingeholt. Bei der Auswahl der Schulen wurde ein Pool aller in Innsbruck vorhandenen Schultypen generiert, aus denen nach dem Zufallsprinzip 38 Schulklassen gezogen wurden. Die Auswahl der Schulklassen basierte auf einer Berechnung der optimalen Stichprobengröße sowie der Stratifizierung der verschiedenen Schulstufen. Anschließend wurde die Zustimmung der einzelnen Schulleiter eingeholt. Es wurde das Einverständnis der SchülerInnen und Eltern eingeholt, an der Studie teilzunehmen.

Die TeilnehmerInnen wurden in der Auswertung inkludiert (a) wenn die Teilnahme an der Untersuchung von den Eltern nicht untersagt wurde, (b) ein Alter von 20 Jahren nicht überschritten wurde und (c) sowohl die soziodemographischen als auch die Fragebogendaten vorhanden waren.

\section{Untersuchungsinstrumente}

\section{Compulsive Internet Use Scale (CIUS)}

Die CIUS ist ein Selbstbeurteilungsverfahren, welches anhand von 14 Items Merkmale der Internetabhängigkeit erfasst. Die Items wurden in Anlehnung an die im DSM-4 definierte Kriterien von substanzgebundener Sucht und pathologischem Glücksspiel entwickelt, und können mit einem fünfstufigen Antwortformat beantwortet werden. Dabei können zwischen 0 und 
56 Punkte erreicht werden. Von den Entwicklern wird der Cut-Off Wert von 28 empfohlen, um pathologischen oder suchtartigen Internetgebrauch zu identifizieren [26]. Mittels eines Cut-Off Wertes von 21 Punkten kann laut Guertler et al. [27] die Prävalenz von problematischem Internetgebrauch am besten geschätzt werden. Das Verfahren wurde in mehreren Teilstichproben entwickelt und weist eine gute Reliabilität ( $\alpha=$ 0,89 ) und Validität auf, ist ökonomisch einsetzbar und liegt in mehreren Sprachen vor [26].

\section{Skala zum Computerspielverhalten (CSV-S)}

Die CSV-S ist ein Selbsteinschätzungsinstrument mit einem fünfstufigen Antwortformat und dient der Erfassung der suchtartigen Nutzung von Computerspielen. Als Computerspiele werden in der CSV-S Konsolenspiele, PC-Spiele, Browser-Games sowie „Andere“ aufgeführt. Das Spielen mit dem Smartphone wird nicht gesondert erfasst, sondern zählt ebenfalls als „Computerspiel“, wodurch eine relativ breite Definition dieses Begriffs gewählt wird. Die Skala orientiert sich inhaltlich an adaptierten Kriterien der substanzgebundenen Abhängigkeitserkrankungen und des pathologischen Glückspiels und erfasst quantitative und qualitative Nutzungsmuster sowie emotionsregulative Aspekte von Computerspielverhalten. Testtheoretische Hintergründe sowie Instruktionen zum gewichteten Scoring finden sich bei Wölfling et al. [28]. Das Computerspielverhalten der SchülerInnen kann in exzessive Nutzung mit einem Cut-Off von 4 Punkten (eine Standardabweichung über dem Durchschnitt) sowie suchtartiges Spielverhalten mit einem Cut-Off von 7 Punkten (zwei Standardabweichungen über dem Durchschnitt) eingeteilt werden. Für die CSV$S$ wurden zufriedenstellende psychometrische Eigenschaften $(\alpha=0,86)$ berichtet [28].

\section{Skala zur allgemeinen Selbstwirksamkeitserwartung} (SWE)

Die eindimensionale Skala SWE erfasst mittels 10 Items die allgemeine optimistische Selbstüberzeugung bzw. Kompetenzerwartung auf einer vierstufigen Skala. Aus der Skala kann ein Gesamtwert durch die Addition der einzelnen Items errechnet werden, wobei Werte zwischen 10-40 Punkten erreicht werden können. Die Mittelwerte liegen in repräsentativen Stichproben bei 29 Punkten $(S D=4,0)$. Die Skala wurde in mehr als 25 Sprachen übersetzt. Die SWE weist durchwegs sehr zufriedenstellende psychometrische Kennwerte $(\alpha=$ $0,80-0,90)$ auf $[29,30]$.

\section{Strukturiertes Interview}

Die strukturierten Interviews wurde auf Grundlage eines früheren Forschungsprojektes [31] für die vorliegende Studie adaptiert und umfasste 71 Fragen zur Peer-Group und Freizeitgestaltung, Substanzgebrauch sowie Fragen zum Internet- und Computerspielverhalten. Die Interviews wurden von geschulten fortgeschrittenen Psychologie-StudentInnen durchgeführt, und dauerten im Durchschnitt 20 min. Die Erhebungen fanden in separaten Räumen statt, und die Daten wurden in anonymisierter Form gesammelt. Die Auswertung der Interviews erfolgte durch die quantitative Analyse der vorgegebenen Antwortmöglichkeiten.

\section{Nutzungsfrequenz}

Für eine Unterteilung in breiter gefasste Kategorien wurde die Stichprobe in Jugendliche mit regelmäßigem Computerspielgebrauch (RU_G) sowie regelmäßigem Gebrauch von Internet (RU_I) unterteilt. In Anlehnung an Batthany et al. (2009) wurden als regelmäßige Gamer (RU_G) diejenigen erfasst, welche an mindestens drei Tagen in der Woche Computerspiele spielten, wohingegen als regelmäßige Internet-User (RU_I) diejenigen Jugendlichen bezeichnet wurden, die angaben, täglich online zu sein. Zusätzlich wurde noch eine Kategorie von Intensivnutzern (RU_I + RU_G) bestimmt, also Jugendliche, die sowohl Internet als auch Computerspiele intensiv nutzen. Diese Unterteilung wurde getroffen, um Intensivnutzer $\mathrm{zu}$ beschreiben, was dem tatsächlichen Medienverhalten von Jugendlichen angesichts bisher publizierter Nutzungsdaten $[1,4]$ entgegenkommt.

\section{Statistische Auswertung}

Die statistische Auswertung erfolgte mit SPSS für Windows (Version 22.0). Die Mittelwerte, Standardabweichungen und prozentuellen Verteilungen für die Verwendung moderner Technologie, Internetabhängigkeit, Computerspielverhalten und Selbstwirksamkeit der SchülerInnen wurden für die Gesamtstichprobe sowie für die Geschlechtergruppen getrennt dargestellt. Gruppenunterschiede bei nominal skalierten Merkmalen wurden mittels Chi-Quadrat-Test berechnet. Unterschiede zwischen zwei Gruppen bei Merkmalen auf intervallskaliertem Datenniveau wurden mittels T-Test für unabhängige Stichproben berechnet. Der Vergleich der Altersgruppen, die Unterschiede hinsichtlich der pathologischen Mediennutzung sowie der Vergleich der Selbstwirksamkeit zwischen den auffälligen und unauffälligen NutzerInnen erfolgte mittels einfacher Varianzanalyse (ANOVA). Bei der Berechnung multipler Gruppenvergleiche wurde der Alpha-Fehler mittels Bonferroni-Korrektur kontrolliert. Das Signifikanzniveau wurde auf $p<0,05$ festgesetzt.

\section{Ergebnisse}

\section{Stichprobe}

\section{Soziodemographische Daten}

Die ursprüngliche Stichprobe umfasste 588 SchülerInnen. Da aufgrund des zeitlichen Aufwands eine Genehmigung für die Interviews von Seiten der Schuldirektoren für einige Schulen nicht erteilt wurde, mussten 187 SchülerInnen aus den Berechnungen ausge- 
Tab. 2 Soziodemographische Daten

\begin{tabular}{|c|c|c|}
\hline \multicolumn{3}{|l|}{ Alter } \\
\hline Mittelwert Alter (SD) & 15,3 & $(2,3)$ \\
\hline 10-14 Jahre & 124 & $31,9 \%$ \\
\hline 14-17 Jahre & 210 & $54,0 \%$ \\
\hline 17-20 Jahre & 55 & $14,1 \%$ \\
\hline \multicolumn{3}{|l|}{ Geschlecht } \\
\hline Männlich & 256 & $65,8 \%$ \\
\hline Weiblich & 133 & $34,2 \%$ \\
\hline \multicolumn{3}{|l|}{ Schultyp } \\
\hline Sonderschule & 26 & $6,7 \%$ \\
\hline Mittelschule/Hauptschule & 77 & $19,8 \%$ \\
\hline Polytechnikum & 18 & $4,6 \%$ \\
\hline Berufsschule & 66 & $17,0 \%$ \\
\hline Gastronomie-Fachschule & 15 & $3,9 \%$ \\
\hline HBLA & 44 & $11,3 \%$ \\
\hline HTL & 88 & $22,6 \%$ \\
\hline Gymnasium & 20 & $5,1 \%$ \\
\hline Fehlend & 35 & $9,0 \%$ \\
\hline \multicolumn{3}{|l|}{ Wohnsituation } \\
\hline Außerhalb der Familie & 21 & $5,4 \%$ \\
\hline Bei einem Elternteil & 86 & $22,1 \%$ \\
\hline Bei beiden Elternteilen & 282 & $72,5 \%$ \\
\hline \multicolumn{3}{|l|}{ Geschwister } \\
\hline Keine & 33 & $8,5 \%$ \\
\hline 1 & 185 & $47,6 \%$ \\
\hline $2-3$ & 138 & $35,5 \%$ \\
\hline$\geq 4$ & 19 & $5,0 \%$ \\
\hline Fehlend & 14 & $3,6 \%$ \\
\hline
\end{tabular}

schlossen werden, da die soziodemographischen Daten nicht vorlagen. Weitere 12 SchülerInnen wurden ausgeschlossen, da sie älter als 20 Jahre waren. Die verbleibenden $n=389$ SchülerInnen (66\%) wurden in die Untersuchung eingeschlossen.

Das mittlere Alter lag bei 15,3 Jahren ( $\pm 2,3$ Jahre), $65,8 \%$ der TeilnehmerInnen waren männlich und 8,5 \% waren Einzelkinder. Ein Großteil der Stichprobe (72,5\%) lebte bei beiden Elternteilen. Die häufigsten Schultypen waren die Höhere Technische Lehranstalt (HTL: 22,6\%) und Mittelschule/Hauptschule (19,8\%). Weitere Details finden sich in Tab. 2.

\section{Verwendung moderner Technologien: Frequenz und Geschlechtsunterschiede (Tab. 3)}

$100 \%$ aller SchülerInnen gaben an einen PC oder Laptop zu benutzen, wobei männliche Studienteilnehmer signifikant häufiger angaben, einen eigenen PC/ Laptop zu verwenden. Während mehr Schüler angaben, eine Spielkonsole zu verwenden, war die Verwendung von Handys und Smartphones unter den Schülerinnen verbreiteter. Insgesamt nutzen $100 \%$ der weiblichen sowie 96,1 \% der männlichen Schüler ein Mobiltelefon bzw. Smartphone. Von den 3,9\% männli- chen Schülern, die kein Smartphone benutzten, waren $54 \%$ Sonderschüler.

Nahezu alle befragten Schüler (96\%) nutzen das Internet mindestens einmal pro Woche, ca. $80 \%$ der befragten Schüler gaben eine Nutzung an mindestens 5 Tagen in der Woche an. Etwa $70 \%$ der befragten Schüler führten an, mindestens einmal pro Woche Computerspiele zu spielen. Hochsignifikante Unterschiede in der Nutzungsfrequenz von Internet und Computerspielen zeigten sich bei getrennter Auswertung nach Geschlechtern. Zusammenfassend zeigte sich, dass der Bereich Computerspiele durchgehend männlich dominiert ist: während 16,3\% der befragten Mädchen angaben, nie ein Computerspiel $\mathrm{zu}$ benutzen, lag die Häufigkeit dieser Antwort bei den männlichen Befragten lediglich bei 3,6\%. Sowohl Intensivnutzer $(>5 \times /$ Woche) als auch fallweise Nutzer von Computerspielen waren konsekutiv signifikant häufiger Jungen $(p<0,001)$. Eine etwas andere Geschlechterverteilung zeigte sich im Bereich InternetNutzung: hier ist lediglich in der Gruppe der häufigen Internet-Nutzer $(>5 \times /$ Woche) der Anteil der männlichen Jugendlichen signifikant größer, während weibliche Jugendliche in den Gruppen mit niederfrequenter Internet-Nutzung häufiger vertreten sind.

\section{Art und soziale Funktion des Internet-Gebrauchs}

Die SchülerInnen nutzten das Internet am häufigsten, um Videos anzusehen $(93.6 \%)$ und um in sozialen Netzwerken online $\mathrm{zu}$ sein $(85 \%)$. Weitere Nutzungsarten waren das „Erledigen von Schularbeiten“ (77 \%) sowie Kommunikation mittels Email (76 \%). Der größte Geschlechtsunterschied zeigte sich bei Internet-Pornografie: $40 \%$ der Jungen, aber nur $2 \%$ der Mädchen gaben an, das Internet für den Besuch von Seiten mit sexuellem Inhalt zu nutzen (für Details siehe Tab. 4).

Für einen Teil der SchülerInnen erleichtert das Internet soziale Aspekte: $35 \%$ finden es leichter im Internet sie selbst zu sein, 40,3\% reden im Internet über andere Dinge als offline und immerhin 20,8\% sprechen im Internet über Dinge, die sie sonst nie ansprechen.

\section{Aufschlüsselung nach Häufigkeit der Mediennutzung}

Aus der Gesamtstichprobe konnten 28,3\% der SchülerInnen als regelmäßige ComputerspielerInnen (RU_G) und 79,2\% als regelmäßige Internetuser (RU_I) identifiziert werden. Sowohl in der Gruppe der RU_G $(36,4 \%$ vs. $14,6 \% ; p<0,001)$ als auch der RU_I $(79,8 \%$ vs. $63,4 \% ; p<0,001)$ fanden sich signifikant mehr männliche als weibliche Jugendliche. Es zeigte sich eine signifikante Überschneidung zwischen den beiden regelmäßigen Usergruppen ( $p=0.02)$, sodass $24,4 \%$ der SchülerInnen der Gruppe der IntensivnutzerInnen zugeordnet werden konnten. Im folgenden werden die SchülerInnen der Intensivnutzergruppe mit den restlichen SchülerInnen verglichen. 
Tab. 3 Verwendung moderner Technologien, getrennt nach Geschlecht

\begin{tabular}{|c|c|c|c|c|c|}
\hline Verwendete Technologien & Gesamt $(n=389)$ & Jungen $(n=256)$ & Mädchen $(n=133)$ & $\mathrm{Chi}^{2}$ & $p$-Wert (zweiseitig) \\
\hline Eigener PC/Laptop & $308(79,4 \%)$ & $214(83,9 \%)$ & $94(70,7 \%)$ & 9,37 & 0,002 \\
\hline Gemeinsamer PC/Laptop & $173(44,5 \%)$ & $97(36,9 \%)$ & $76(57,1 \%)$ & 13,14 & $<0,001$ \\
\hline TV & $353(91,0 \%)$ & $231(90,6 \%)$ & $122(91,7 \%)$ & 0,14 & 0,71 \\
\hline Handy/Smartphone & $378(97,4 \%)$ & $245(96,1 \%)$ & $133(100,0 \%)$ & 5,88 & 0,02 \\
\hline Spielekonsole & $264(68,6 \%)$ & $185(73,1 \%)$ & $79(59,8 \%)$ & 7,09 & 0,01 \\
\hline \multicolumn{6}{|l|}{ Häufigkeit Spiele } \\
\hline Nie & $29(7,7 \%)$ & $9(3,6 \%)$ & $20(16,3 \%)$ & \multirow[t]{5}{*}{44,43} & \multirow[t]{5}{*}{$<0,001$} \\
\hline$<1 \mathrm{mal}$ im Monat & $41(10,9 \%)$ & $16(6,3 \%)$ & $25(20,3 \%)$ & & \\
\hline 1-3 mal im Monat & $50(12,9 \%)$ & $32(12,5 \%)$ & $18(13,5 \%)$ & & \\
\hline 1-3 mal pro Woche & $159(40,9 \%)$ & $107(41,8 \%)$ & $52(39,1 \%)$ & & \\
\hline$>5$ mal pro Woche & $110(28,3 \%)$ & $92(35,9 \%)$ & $18(13,5 \%)$ & & \\
\hline \multicolumn{6}{|l|}{ Häufigkeit Internetkonsum } \\
\hline$<1$ mal im Monat & $6(1,5 \%)$ & $2(0,8 \%)$ & $4(3,0 \%)$ & \multirow[t]{4}{*}{10,21} & \multirow[t]{4}{*}{0,02} \\
\hline 1-3 mal im Monat & $9(2,3 \%)$ & $4(1,6 \%)$ & $5(3,8 \%)$ & & \\
\hline 1-3 mal pro Woche & $66(17,0 \%)$ & $36(14,1 \%)$ & $30(22,6 \%)$ & & \\
\hline$>5$ mal pro Woche & $308(79,2 \%)$ & 214 (83,6 \%) & 94 (70,7 \%) & & \\
\hline
\end{tabular}

Tab. 4 Art der Internet-Nutzung (Prozentangaben betreffen Gesamtstichprobe, Mehrfachnennungen möglich)

\begin{tabular}{|l|l|l|l|l|l|}
\hline Art der Internet-Nutzung & Häufigkeit Gesamt in \% & Häufigkeit Jungen in \% & Häufigkeit Mädchen in \% & Chi(2) & $p$ \\
\hline Ansehen von Videos & 93,6 & 96,5 & 88,0 & 10,55 & 0,001 \\
\hline Soziale Netzwerke & 85,1 & 85,9 & 83,5 & 0,42 & 0,51 \\
\hline Erledigen von Schularbeiten & 77,1 & 77,7 & 75,9 & 0,16 & 0,69 \\
\hline Email & 75,8 & 82,0 & 65,2 & 13,56 & $<0,001$ \\
\hline Herunterladen von Musik/Filmen & 65,8 & 72,8 & 53,4 & 14,75 & $<0,001$ \\
\hline Online-Kauf & 47,1 & 51,6 & 22,7 & 29,73 & $<0,001$ \\
\hline Chatrooms & 33,9 & 36,6 & 29,5 & 1,93 & 0,17 \\
\hline Sex-Seiten & 26,0 & 40,1 & 1,6 & 64,04 & $<0,001$ \\
\hline Virtuelle Räume (bspw. WOW) & 11,6 & 12,6 & 9,8 & 0,64 & 0,43 \\
\hline
\end{tabular}

Die IntensivnutzerInnen hatten im selben Umfang Menschen in ihrer Umgebung, denen sie voll vertrauen konnten. Allerdings berichteten sie signifikant häufiger, im Internet leichter sie selbst sein zu können $(p<0,01)$ und dort auch über andere Dinge sprechen zu können $(p<0,001)$. Bezüglich der Nutzungsinhalte gaben die IntensivnutzerInnen häufiger an Dinge $\mathrm{zu}$ kaufen $(p=0,02)$, Sexseiten zu besuchen $(p<0,001)$ und E-Mails zu schreiben/lesen $(p=0,05)$.

\section{Prävalenz von pathologischem Mediengebrauch}

\section{Suchtartiger Gebrauch von Internet}

Für 3,3\% der Stichprobe wurde pathologisches oder suchtartiges Nutzungsverhalten, sowie für 7,7\% der Stichprobe ein problematisches Internetnutzungsverhalten gefunden. Weder für den CIUS Gesamtscore noch für die Subskalen zeigten sich signifikante Geschlechtsunterschiede. In den folgenden Subanalysen werden SchülerInnen mit unauffälligem, problematischem und suchtartigem Internetnutzungsverhalten (NV) verglichen.

Es fanden sich kaum Unterschiede hinsichtlich der Verwendungsinhalte des Internets. Lediglich in Be- zug auf die Verwendung von Chatrooms $(p=0,05)$ und ob Zeit in virtuellen Räumen (bspw. WOW) verbracht wird $(p<0,001)$ fand sich ein Unterschied zwischen den Gruppen (unauffälliges $\mathrm{NV}<$ problematisches NV < suchtartiges NV).

SchülerInnen mit problematischen oder suchtartigem NV gaben jedoch auch signifikant häufiger als unauffällige SchülerInnen an, dass es leichter für sie sei im Internet sie selbst sein zu können $(p<0,001)$ und dass sie online über andere Dinge reden würden $(p=0,01)$, bzw. über persönliche Dinge sprechen zu können, die sie sonst nicht ansprechen könnten $(p=0,001)$. Sie würden auch häufiger nicht wissen, was sie mit ihrer Freizeit anfangen könnten ( $p=$ $0,01)$. Hinsichtlich der subjektiven Selbstwirksamkeit zeigten unauffällige SchülerInnen signifikant bessere Werte als SchülerInnen mit problematischen NV ( $F=$ $4,1 ; p=0,02)$. Obwohl die Werte der unauffälligen SchülerInnen vergleichbar mit den SchülerInnen mit suchtartigem NV waren (29,7 vs. 29,9), unterschieden sich letztere jedoch nicht signifikant von den SchülerInnen mit problematischen NV, wahrscheinlich aufgrund des geringen $N$. 
Tab. 5 Geschlechterspezifische Darstellung von CSV-S, CIUS und SWE

\begin{tabular}{|c|c|c|c|c|c|}
\hline & Gesamt $(n=389)$ & Jungen $(n=256)$ & Mädchen $(n=133)$ & Teststatistik & $p$-Wert \\
\hline$C S V-S^{a}$ & $1,5(2,8)$ & $1,9(2,9)$ & $0,7(2,4)$ & $t=6,80$ & $<0,001$ \\
\hline Unauffällige Nutzung & $338(86,9 \%)$ & $212(82,8 \%)$ & $126(94,7 \%)$ & \multirow[t]{3}{*}{$C h I^{2}=11,2$} & \multirow[t]{3}{*}{0,004} \\
\hline Exzessive Nutzung & $30(7,7 \%)$ & $25(9,8 \%)$ & $5(3,8 \%)$ & & \\
\hline Suchtartige Nutzung & $21(5,4 \%)$ & $19(7,4 \%)$ & $2(1,5 \%)$ & & \\
\hline CIUS Gesamtscore & $11,15(8,0)$ & $11,4(7,8)$ & $10,7(8,3)$ & $t=0,82$ & 0,42 \\
\hline Unauffällige Internetnutzung & $341(88,8 \%)$ & $224(88,9 \%)$ & $117(88,6 \%)$ & \multirow[t]{3}{*}{ Chi $=0,11$} & \multirow[t]{3}{*}{0,95} \\
\hline Problematische Internetnutzung & $30(7,7 \%)$ & $20(7,9 \%)$ & $10(7,6 \%)$ & & \\
\hline Suchtartige Internetnutzung & $13(3,3 \%)$ & $8(3,2 \%)$ & $5(3,7 \%)$ & & \\
\hline Selbstwirksamkeit & $29,4(5,3)$ & $30,1(5,0)$ & $28,1(5,4)$ & $t=3,59$ & $<0,001$ \\
\hline
\end{tabular}

Ein Kreuzvergleich zeigte hochsignifikante Überschneidungen zwischen SchülerInnen mit auffälligem Computerspielverhalten und auffälliger Internetnutzung ( $p<0,001)$, die $2,1 \%$ der Stichprobe ausmachten.

\section{Suchtartiger Gebrauch von Computerspielen}

In der vorliegenden Stichprobe zeigten sich mit einem Mittelwert von 1,6 (SD = 2,8) fast idente Werte zur Normstichprobe von Wölfling et al. [28], was für die Repräsentativität der vorliegenden Stichprobe spricht. Insgesamt berichteten $7,7 \%$ der Gesamtstichprobe ein exzessives und $5,4 \%$ ein suchtartiges Computerspielverhalten. Es zeigte sich ein klarer Geschlechtsunterschied $(p<0,001)$, wobei signifikant mehr männliche als weibliche UntersuchungsteilnehmerInnen ein exzessives bzw. suchtartiges Computerspielverhalten angaben. In den folgenden Subanalysen werden Schüler und Schülerinnen mit suchtartigem und unauffälligem Computerspielverhalten verglichen.

SchülerInnen mit suchtartigem Computerspielverhalten würden ihre Freizeit häufiger alleine verbringen ( $p=0,013)$ und wüssten öfter nicht, was sie mit ihrer Freizeit anfangen sollen $(p=0,02)$. Bezüglich dem Freizeitverhalten und der Selbstwirksamkeit konnten keine Unterschiede zwischen den Gruppen festgestellt werden. Auch zwischen verschiedenen Altersgruppen zeigte sich kein signifikanter Unterschied hinsichtlich des suchtartigen Computerspielverhaltens $(F=1,6 ; p=$ $0,19)$. SchülerInnen mit suchtartigem Computerspielverhalten gaben signifikant häufiger an, sich nicht an die FSK-Altersvorgaben der PC-Spiele zu halten ( $p=$ $0,05)$, wobei darauf hingewiesen werden muss, dass auch in der Gesamtstichprobe $70 \%$ der SchülerInnen angab, sich nicht an die FSK-Vorgaben zu halten. Tab. 5 zeigt für die Instrumente CSV-S, CIUS und SWE Mittelwerte sowie eine Aufschlüsselung nach dysfunktionalen Nutzungsmustern, jeweils für die Gesamtstichprobe sowie nach Geschlechtern getrennt. Signifikanzniveaus sowie die verwendete Teststatistik sind den letzten zwei Spalten rechts zu entnehmen.

\section{Diskussion}

Ziel der vorliegenden Studie war die Erhebung repräsentativer epidemiologischer Daten zur Computerspiel- und Internetnutzung von Jugendlichen in Tirol (Österreich). Anhand der Untersuchung sollten die Häufigkeit, Art und Intensität der Internet- und Computerspielnutzung von Tiroler SchülerInnen abgebildet werden, um einen Vergleich zu bereits in der Literatur bestehenden Prävalenzzahlen zu ermöglichen. Darüber hinaus galt es, Unterschiede zwischen Schülern mit einem pathologischen Internet- und Computerspiel-Nutzungsmuster und Schülern, welche ein unauffälliges Nutzungsmuster aufweisen, abzubilden.

Wie in vergleichbaren Nutzungsstudien aus dem deutschen Sprachraum (bspw. [1]) zeigte sich auch in unserer Stichprobe eine Nutzungsrate von nahezu 100 \% für PCs/Laptops sowie für Smartphones. 96,2 \% unserer befragten Jugendlichen benutzten zumindest einmal wöchentlich das Internet, ca. $80 \%$ an mehr als 5 Tagen in der Woche. Somit kann auch in unserer Erhebung die praktisch vollständige Durchdringung einer jugendlichen Kohorte mit einschlägiger Technik bzw. Internet-Nutzung dargestellt werden.

In unserer Studie zeigten 7,7 \% der untersuchten Jugendlichen Hinweise auf problematischen sowie 3,3\% der Jugendlichen Hinweise auf pathologischen bzw. suchtartigen Internet-Gebrauch. Damit liegen unsere Ergebnisse etwas unter den Durchschnittswerten der SEYLE-Studie [11], in der $13,5 \%$ der untersuchten Jugendlichen Hinweise auf problematischen („maladaptiven“) Internet-Gebrauch sowie 4,4\% Hinweise auf pathologischen bzw. suchtartigen Internet-Gebrauch zeigten. Es zeigt sich jedoch eine fast deckungsgleiche Übereinstimmung mit der österreichischen Substichprobe der SEYLE-Studie, in der bei 3,1\% der Untersuchten suchtartiger Internetgebrauch gemessen werden konnte.

13,1 \% unserer Stichprobe zeigten auffälliges Computerspiel-Verhalten, wobei 7,7\% über exzessives und $5,4 \%$ über suchtartiges Computerspielverhalten berichteten. Unsere Studie zeigte einen statistisch hochsignifikanten Geschlechtsunterschied dahingehend, 
dass weitaus mehr männliche als weibliche UntersuchungsteilnehmerInnen exzessives bzw. suchtartiges Computerspielverhalten angaben. Damit stehen unsere Daten in guter Übereinstimmung mit einer vorhergegangenen Studie an Wiener Jugendlichen mit denselben Untersuchungsinstrumenten, in der insgesamt $12,3 \%$ der untersuchten Jugendlichen problematisches Computerspiel-Verhalten zeigten (9,6\% exzessives und 2,7\% suchtartiges Spielverhalten) [25]. Auch bezüglich der Geschlechtsunterschiede hinsichtlich der Ausprägung des problematischen Computerspielens fanden Batthyány et al. [25] vergleichbare Ergebnisse.

Internet-Gaming-Disorder (IGD) nach DSM-5, also eine sich möglicherweise additiv verstärkende $\mathrm{Ab}$ hängigkeitsproblematik bezüglich Internet und Computerspielen, wurde bisher in europäischen Studien wenig untersucht, Müller et al. [18] ermittelten in der einzig verfügbaren Studie eine vergleichsweise niedrige Prävalenzrate von $1,6 \%$. In unserer Studie lag der Anteil der Jugendlichen mit abhängigem Onlineund abhängigem Computerspiel-Verhalten bei 2,1\%, wobei wissenschaftlich nicht untersucht ist, ob diese Kombination eine grobe Annäherung an IGD darstellen könnte.

In den qualitativen Erhebungen gaben suchtartige Gamer unserer Stichprobe signifikant häufiger an, ihre Freizeit alleine zu verbringen bzw. nicht zu wissen, was sie mit ihrer Freizeit anfangen sollen. Jugendliche mit PGI gaben signifikant häufiger an, dass es leichter für sie sei im Internet „sie selbst sein zu können“, und dass sie online über andere Dinge reden würden, bzw. über persönliche Dinge sprechen zu können, die sie sonst nicht ansprechen könnten. Sie würden auch häufiger nicht wissen, was sie mit ihrer Freizeit anfangen könnten und schätzen ihre Selbstwirksamkeit geringer ein als SchülerInnen ohne PGI. Diese Befunde stehen in guter Übereinstimmung mit einer Reihe von Vorbefunden, die soziale Eingebundenheit sowie breit aufgestellte außerschulische Aktivitäten bzw. Freizeitaktivitäten als Schutzfaktoren gegen pathologischen Mediengebrauch identifizierten [32, 33]. Umgekehrt scheinen Langeweile sowie Einsamkeit die Wahrscheinlichkeit des Auftretens einer pathologischen Mediennutzung zu begünstigen $[17,32,34]$.

Zusätzlich zu „Pathologie-Kriterien“ haben wir eine Gruppe von „Intensivnutzern“ (regelmäßige Gamer und Internetnutzer) erhoben. Die 24,4 \% Intensivnutzer unserer Stichprobe waren überwiegend männlich und gaben im Vergleich zu durchschnittlichen Nutzern signifikant häufiger an, im Internet leichter sie selbst sein zu können und dort auch über wichtige Dinge sprechen zu können. Diese Befunde decken sich mit einer rezenten Arbeit von Strittmatter et al. [23], in der die AutorInnen ebenfalls männliche Jugendliche mit Schwierigkeiten in der peer-group als diejenigen identifizierten, welche problematischen
Internetkonsum kombiniert mit problematischem Computerspiel-Verhalten zeigten.

Erwähnenswert scheinen große Geschlechtsunterschiede der jugendlichen ProbandInnen im Bereich Computerspiele sowie bei der qualitativen Nutzungserhebung. Im Einklang mit einer Reihe von bisherigen Erhebungen [25, 32, 35-37] zeigten sich auch in unserer Studie männliche Jugendliche hochsignifikant häufiger von problematischem ComputerspielGebrauch betroffen als ihre weiblichen Altersgenossinnen. Der größte Signifikanzunterschied bei der Erhebung der Internet-Nutzungsarten zeigte sich im Bereich der Pornografie: 40,1\% der Jungen aber nur 1,6 \% der Mädchen gaben an, das Internet für den Besuch von Seiten mit sexuellem Inhalt zu nutzen. Auch dieser Befund unserer Studie deckt sich mit bisher erhobenen Daten [38].

Limitierend für die Generalisierbarkeit unserer Befunde ist die Überrepräsentiertheit von männlichen Jugendlichen $(65,8 \%)$ in unserem Sample, wodurch sich Prävalenzzahlen für PGI und vor allem PGC möglichweise nach oben verfälschen. Des Weiteren könnte die Repräsentativität der Stichprobe aufgrund der vergleichsweise geringen Anzahl von Gymnasialklassen sowie des hohen Anteils technischer Schulen beeinträchtigt sein. Aufgrund der geringen Anzahl der Jugendlichen mit suchtartigem Internetnutzungsverhalten sind die Gruppenvergleiche mit dieser Substichprobe nur in begrenztem Maße aussagekräftig. Die Studie wurde als nicht-klinische Feldstudie konzipiert, wodurch uns keine Informationen zu eventuell vorliegenden komorbiden psychischen Störungen sowie dem Intelligenzniveau zur Verfügung standen. Seit der Durchführung der Studie 2011/2012 erfolgten technische Innovationen, die einen noch intensiveren Gebrauch des Internet vor allem mittels mobiler Endgeräte ermöglichten [4]. Außerdem fällt in diese Zeit die rasante Verbreitung des Gebrauchs von Messenger-Diensten [4], die in unserer Erhebung noch nicht explizit abgefragt wurde. Somit könnten aktuelle Internet-Nutzungsraten von Jugendlichen mittels Smartphone oder Tablet nochmals deutlich höher liegen als in unserer Erhebung erfasst.

\section{Fazit}

Zusammenfassend kann mit unserer Studie die Übertragbarkeit von weltweit gewonnenen Daten zur Häufigkeit von pathologischem Mediengebrauch auf Tiroler Jugendliche gezeigt werden. Neben dem Ansehen von Videos sowie Aktivitäten in sozialen Netzwerken waren in unserer Studie das Verfassen von Schularbeiten sowie die Kommunikation mittels Email die häufigsten Gründe, online zu gehen. Online-Aktivitäten spielen für Jugendliche somit eine wichtige Rolle in der Auseinandersetzung mit Gleichaltrigen in der peer-group, sind aber auch in Schule, Ausbildung und Beruf von essentieller Bedeutung. Eine „Medienabstinenz“ - im Gegensatz zu stoffgebun- 
denen Suchterscheinungen - scheint daher schwer möglich, Internet-,Verbote“ sind im Zeitalter von flächendeckendem WLAN Jugendlichen gegenüber nicht durchsetzbar. Präventive medienpädagogische Maßnahmen müssten diese Tatsache berücksichtigen, ein Heranwachsen von Medienkompetenz ist bei jungen Menschen nur in Form von freiwilligen Vereinbarungen bei gleichzeitig „laufendem Konsum“ möglich. Somit müsste unserer Meinung nach das Erlernen von verantwortungsbewusster Mediennutzung bereits sehr früh Gegenstand von entsprechenden pädagogischen Bausteinen sein, z. B. in Form eines Pflichtfaches ab der 5. Schulstufe. Unsere Ergebnisse weisen darauf hin, dass für einen kompetenten Umgang mit digitalen Medien ein pädagogischer Ansatz nötig sein könnte, der sowohl Gender-spezifische Bedürfnisse als auch entwicklungstypische Aspekte von Gruppenzugehörigkeit und Selbstwahrnehmung berücksichtigt.

Angesichts der in den letzten Jahren replizierten Prävalenzziffern zeigt ein nicht unbedeutender Teil der jugendlichen Bevölkerung Schwierigkeiten, sich kompetente Mediennutzung anzueignen, ein kleinerer Teil von Jugendlichen läuft Gefahr, manifeste Abhängigkeiten von Medienangeboten zu entwickeln. Diesen Entwicklungen wird voraussichtlich auch im ICD-11 mit der Einführung einheitlicher Kriterien Rechnung getragen. Die Verwendung einheitlicher Störungskriterien stellt eine wichtige Grundlage für replizierbare Prävalenzschätzungen sowie für die Entwicklung störungsspezifischer Therapieansätze dar.

\section{Ausblick}

In einem Folgeprojekt wird eine klinische Inanspruchnahme-Population (stationäre Patienten einer Kinderund Jugendpsychiatrie) mit denselben Instrumenten untersucht, um Zusammenhänge zwischen psychopathologischen Phänomenen und pathologischer Mediennutzung näher darzustellen. Weiters soll untersucht werden, ob junge Menschen mit psychischen Erkrankungen womöglich in viel stärkerem Ausmaß von dysfunktionaler Mediennutzung betroffen sein könnten. Die Symptomatik klassischer psychopathologischer Phänomene von Kindern und Jugendlichen könnte sich aktuell vor dem Hintergrund einer digital vernetzten Welt verändert haben, und es wäre die Aufgabe von Fachleuten, darauf zu reagieren.

Danksagung Die Durchführung der Studie wurde durch die Ambulante Suchtprävention der Innsbrucker Sozialen Dienste gefördert.

Open access funding provided by University of Innsbruck and Medical University of Innsbruck.

\section{Einhaltung ethischer Richtlinien}

Interessenkonflikt D. Riedl, A. Stöckl, C. Nussbaumer, G. Rumpold, K. Sevecke und M. Fuchs geben an, dass kein Interessenkonflikt besteht.

Ethische Standards Alle beschriebenen Untersuchungen am Menschen wurden mit Zustimmung der zuständigen EthikKommission, im Einklang mit nationalem Recht sowie gemäß der Deklaration von Helsinki von 1975 (in der aktuellen, überarbeiteten Fassung) durchgeführt. Von allen beteiligten Patienten liegt eine Einverständniserklärung vor.

Open Access Dieser Artikel wird unter der Creative Commons Namensnennung 4.0 International Lizenz (http:// creativecommons.org/licenses/by/4.0/deed.de) veröffentlicht, welche die Nutzung, Vervielfältigung, Bearbeitung, Verbreitung und Wiedergabe in jeglichem Medium und Format erlaubt, sofern Sie den/die ursprünglichen Autor(en) und die Quelle ordnungsgemäß nennen, einen Link zur Creative Commons Lizenz beifügen und angeben, ob Änderungen vorgenommen wurden.

\section{Literatur}

1. Frees B, Koch W. Ergebnisse der ARD/ZDF-Onlinestudie 2015. Internetnutzung: Frequenz und Vielfalt nehmen in allen Altersgruppen zu. Media Perspekt. 2015;09:366-77.

2. Statistik Austria. Europäische Erhebung über den IKT-Einsatzin Haushalten 20152015. http://www.statistik.at/web_ de/statistiken/energie_umwelt_innovation_mobilitaet/ informationsgesellschaft/ikt-einsatz_in_haushalten/ index.html.Zugegriffen: 02. Jun 2016.

3. Van Eimeren B, Frees B. Ergebnisse der ARD/ZDF-Onlinestudie 2013-Rasanter Anstieg des Internetkonsums. Media Perspekt. 2013;44:358-72.

4. Koch W, Frees B. Ergebnisse der ARD/ZDF-Onlinestudie 2015. Unterwegsnutzung des Internets wächst bei geringerer Intensität. Media Perspekt. 2015;09:378-82.

5. Fuchs M, Kemmler G, Steiner H, Marksteiner J, Haring C, Miller C, et al. Child and adolescent psychiatry patients coming of age: a retrospective longitudinal study of inpatient treatment in Tyrol. BMC Psychiatry. 2016;16:225. doi:10.1186/s12888-016-0910-x.

6. PhilippJ,ZeilerM, Waldherr K, Nitsch M, DurW, KarwautzA, et al. The Mental Health in Austrian Teenagers (MHAT)-Study: preliminary results from a pilot study. Neuropsychiatr. 2014;28(4):198-207. doi:10.1007/s40211-014-0131-9.

7. Dowling NA, Quirk KL. Screening for internet dependence: do the proposed diagnostic criteria differentiate normal from dependent internet use? Cyberpsychol Behav. 2009;12(1):21-7.

8. Bauernhofer K, Papousek I, Fink A, Unterrainer HF, Weiss EM. Problematic internet use (PIN) - a review of assessment questionnaires and risk factors. Neuropsychiatr. 2016;30(1):2-9. doi:10.1007/s40211-015-0169-3.

9. American Psychiatric Association. DSM-5. Diagnostic and statistical manual of mental disorders, 5. Aufl. Arlington: APA;2013.

10. Mann K, Fauth-Buhler M, Seiferth N, Heinz A. The concept of behavioral addiction and limits of the term addiction. Nervenarzt. 2013;84(5):548-56.

11. Durkee T, Kaess M, Carli V, Parzer P, Wasserman C, Floderus $B$, et al. Prevalence of pathological internet use among adolescents in Europe: Demographic and social factors. Addiction. 2012;107(12):2210-22. 
12. Kaess M, Durkee T, Brunner R, Carli V, Parzer P, Wasserman C, et al. Pathological Internet use among European adolescents: psychopathology and self-destructive behaviours. Eur Child Adolesc Psychiatry. 2014;23(11):1093-102.

13. van den Eijnden RJJM, Meerkerk G-J, Vermulst AA, Spijkerman R, Engels RCME. Online communication, compulsive internet use, and psychosocial well-being among adolescents: a longitudinal study. Dev Psychol. 2008;44(3):655-65.

14. van Rooij AJ, Schoenmakers TM, van de Eijnden RJM, van de Mheen D. Compulsive Internet use: the role of online gaming and other Internet applications. J Adolesc Health. 2010;47(1):51-7.

15. Petry J. Pathologisches Glücksspielen und PC-/InternetSpielen. In: AHG PdWd (Hrsg.). Rehabilitation und Alter: Schriftenreihe des Wissenschaftsrates der AHG. Düsseldorf:AHG;2010.S.75-89.

16. Cheng $\mathrm{C}, \mathrm{Li} \mathrm{AY}$. Internet addiction prevalence and quality of (real) life: a meta-analysis of 31 nations across seven world regions. CyberpsycholBehavSoc Netw.2014;17(12):755-60.

17. Cao F, Su L. Internet addiction among Chinese adolescents: prevalence and psychological features. Child Care Health Dev. 2007;33(3):275-81.

18. Kim K, Ryu E, Chon MY, Yeun EJ, Choi SY, Seo JS, et al. Internet addiction in Korean adolescents and its relation to depression and suicidal ideation: a questionnaire survey. Int J Nurs Stud. 2006;43(2):185-92.

19. Moreno MA, Jelenchick L, Cox E, Young H, Christakis DA. Problematic internet use among US youth: a systematic review. Arch Pediatr Adolesc Med. 2011;165(9):797-805.

20. Milani L, Osualdella D, Di Blasio P. Quality of interpersonal relationships and problematic Internet use in adolescence. Cyberpsychol Behav.2009;12(6):681-4.

21. TsitsikaA, Janikian M, SchoenmakersTM, TzavelaEC, Olafsson $\mathrm{K}$, Wojcik S, et al. Internet addictive behavior in adolescence: a cross-sectional study in seven European countries. Cyberpsychol Behav Soc Netw. 2014;17(8):528-35. doi:10.1089/cyber.2013.0382.

22. Blinka L, Škařupová K, Ševčíková A, Wölfling K, Müller KW, Dreier M. Excessive internet use in European adolescents: what determines differences in severity? Int J Public Health 2015;60(2):249-56.

23. Strittmatter E, Kaess M, Parzer P, Fischer G, Carli V, Hoven CW, et al. Pathological internet use among adolescents: Comparing gamers and non-gamers. Psychiatry Res. 2015;228(1):128-35.

24. Müller KW, Janikian M, Dreier M, Wolfling K, Beutel ME, Tzavara C, et al. Regular gaming behavior and internet gaming disorder in European adolescents: results from a cross-national representative survey of prevalence, predictors, and psychopathological correlates. Eur Child Adolesc Psychiatry. 2015;24(5):565-74.

25. Batthyány D, Müller KW, Benker F, Wölfling K. Computer game playing: clinical characteristics of dependence and abuse among adolescents. Wien Klin Wochenschr. 2009;121(15-16):502-9.

26. Meerkerk GJ, Van Den Eijnden RJ, Vermulst AA, Garretsen HF. The Compulsive Internet Use Scale (CIUS): some psychometric properties. Cyberpsychol Behav. 2009;12(1):1-6.

27. Guertler D, Rumpf HJ, Bischof A, Kastirke N, Petersen KU, John U, et al. Assessment of problematic internet use by the compulsive Internet use scale and the internet addiction test: a sample of problematic and pathological gamblers. EurAddict Res. 2014;20(2):75-81.

28. Wölfling K, Müller KW, Beutel M. Reliabilität und Validität der Skala zum Computerspielverhalten (CSV-S). Psychother Psychosom Med Psychol.2011;61(5):216-24.

29. Schwarzer R, Jerusalem M. SkalenzurErfassungvon Lehrerund Schülermerkmalen. Dokumentation der psychometrischen Verfahren im Rahmen der Wissenschaftlichen Begleitung des Modellversuchs Selbstwirksame Schulen. Berlin: Freie Universität Berlin; 1999.

30. Schwarzer R, Mueller J, Greenglass E. Assessment of perceived general self-efficacy on the Internet: Data collection in cyberspace. Anxiety Stress Coping. 1999;12(2):145-61.

31. Rumpold G, Klingseis M, Dornauer K, Kopp M, Doering S, Hofer S, et al. Psychotropic substance abuse among adolescents: a structural equation model on risk and protective factors. Subst Use Misuse. 2006;41(8):1155-69. doi:10.1080/10826080600752136.

32. Kuss DJ, Griffiths MD. Online gaming addiction in children and adolescents: a review of empirical research. J Behav Addict. 2012;1(1):3-22.

33. Wang L, Luo J, Bai Y, Kong J, Luo J, Gao W, et al. Internet addiction of adolescents in China: prevalence, predictors, and association with well-being. Addict Res Theory. 2013;21(1):62-9.

34. Mythily S, Qiu S, Winslow M. Prevalence and correlates of excessive Internet use amongyouth in Singapore. Ann Acad Med Singapore. 2008;37(1):9-14.

35. RehbeinF, Kleimann M, MößleT. Prevalence and riskfactors of video game dependency in adolescence: results of a German nationwide survey. Cyberpsychol Behav Soc Netw. 2010;13(3):269-77.

36. Kiraly O, Griffiths MD, Urban R, Farkas J, Kokonyei G, Elekes $\mathrm{Z}$, et al. Problematic internet use and problematic online gaming are not the same: findings from a large nationally representative adolescent sample. Cyberpsychol BehavSoc Netw. 2014;17(12):749-54.

37. Mentzoni RA, Brunborg GS, Molde H, Myrseth H, Mår Skouverøe KJ, Hetland J, et al. Problematic video game use: estimated prevalence and associations with mental and physical health. Cyberpsychol Behav Soc Netw. 2011;14(10):591-6.

38. Doornwaard SM, van den Eijnden RJ, Baams L, Vanwesenbeeck I, ter Bogt TF. Lower Psychological Well-Being and Excessive Sexual Interest Predict Symptoms of Compulsive Use of Sexually Explicit Internet Material Among Adolescent Boys. JYouth Adolesc. 2016;45(1):73-84. 